\title{
Busca por cuidado em pronto atendimento público pela população feminina
}

Search for care in public emergency services by the female population

\section{Búsqueda de atención en servicios públicos de emergencia por parte de la población femenina}

Recebido: $14 / 06 / 2020$

Aprovado: 28/10/2020

Publicado: 05/11/2020

\author{
Thais Renata Ribeiro de Souza ${ }^{1}$ \\ Denize Jussara Rupolo Dall'Agnol ${ }^{2}$ \\ Pollyanna de Siqueira Queirós ${ }^{3}$ \\ Helga Yuri Doi ${ }^{4}$ \\ Raiane Rocha Xavier ${ }^{5}$ \\ Daniela do Carmo Oliveira Mendes ${ }^{6}$
}

Esta pesquisa teve como objetivo descrever a busca por cuidado em um pronto atendimento de um hospital público por mulheres e a sua relação com o atendimento na atenção básica em saúde. Este é um estudo de abordagem quantitativa do tipo descritivo-transversal, desenvolvido em um município no interior do CentroOeste brasileiro, em 2016, com 181 mulheres. Realizou-se análise descritiva dos dados. 0 perfil sócio demográfico destacou-se: faixa etária entre 36 e 59 anos de idade (38,1\%), casadas (49,8\%), ensino médio completo (27,1\%) e renda menor que dois salários mínimos (39,7\%), afirmaram procurar o pronto atendimento hospitalar por manifestações clínicas relacionadas a doenças infecciosas $(23,2 \%)$, não era a primeira vez que buscava por atendimento nessa instituição $(84,5 \%)$ e que antes de buscar pelo pronto atendimento não esteve em outro serviço de saúde $(64,1 \%)$. Dentre as que buscaram outro serviço de saúde antes de procurar o hospital, a maioria (81,6\%) relatou ter buscado a Unidade Básica de Saúde A efetiva sistematização da rede de atenção à saúde pública se faz importante para delinear práticas de cuidado mais concretas e qualificadas.

Descritores: Saúde Pública; Mulheres; Acesso aos Serviços de Saúde.

This research aimed to describe the search for care in emergency rooms at a public hospital by women and their relationship with primary health care. This is a study with a quantitative approach of the descriptive-transversal type, developed in a municipality in the interior of the Brazilian Midwest region, in 2016, with 181 women. Descriptive analysis of data was performed. The socio-demographic profile stood out: age group between 36 and 59 years old (38.1\%), married (49.8\%), complete high school education $(27.1 \%)$ and income below two minimum wages $(39,7 \%)$, claimed to seek hospital emergency care for clinical manifestations related to infectious diseases (23.2\%), it was not the first time they sought care at this institution (84.5\%) and had not sought care on another healthc (64.1\%). Among those who sought another health service before looking for a hospital, the majority $(81.6 \%)$ reported having sought a basic health unit The effective systematization of the public health care network is important to outline more concrete care practices and qualified.

Descriptors: Public Health; Women; Health Services Accessibility.

Esta investigación tuvo como objetivo describir la búsqueda de atención de emergencia en un hospital público por parte de las mujeres y su relación con la atención básica en salud. Este es un estudio de enfoque cuantitativo de tipo descriptivo-transversal, desarrollado en una ciudad del interior del Centro-Oeste brasileño, en 2016, con 181 mujeres. Se realizó un análisis descriptivo de los datos. Se destacó el perfil sociodemográfico: grupo de edad entre 36 y 59 años $(38,1 \%)$, casadas $(49,8 \%)$, escuela secundaria completa $(27,1 \%)$ e ingresos inferiores a dos salarios mínimos $(39,7 \%)$, afirmaron buscar atención hospitalaria para manifestaciones clínicas relacionadas con enfermedades infecciosas $(23,2 \%)$, no era la primera vez que buscaba atención en esta institución $(84,5 \%)$ y que antes de buscar atención no estuvo en otro servicio de salud $(64,1 \%)$. Entre las que buscaron otro servicio de salud antes de buscar atención hospitalaria, la mayoría $(81,6 \%)$ declaró haber buscado la Unidad Básica de Salud. La sistematización efectiva de la red de atención a la salud pública es importante para diseñar prácticas de atención más concretas y cualificadas.

Descriptores: Salud Pública; Mujeres; Accesibilidad a los Servicios de Salud.

1. Enfermeira. Especialista em Enfermagem do Trabalho. Enfermeira do Hospital de Urgências de Anápolis, G0, Brasil. ORCID: 0000-0002-5385-2616 E-mail: thais_ribeirosouza@hotmail.com

2. Enfermeira. Especialista em Docência do Ensino Superior. Mestre em Imunologia e Parasitologia. Doutora em Farmacologia e Biotecnologia. Professora Adjunta do curso de Enfermagem da Universidade Estadual do Mato Grosso (UNEMAT), Tangará da Serra, MT, Brasil. ORCID: 0000-0002-2551-2382 E-mail: denize.dallagnol@unemat.br

3. Enfermeira. Especialista em Gestão em Saúde. Mestre em Enfermagem. Doutoranda em Enfermagem Psiquiátrica pela Escola de En fermagem de Ribeirão Preto, da Universidade de São Paulo. Professora Assistente do curso de Enfermagem da UNEMAT, Tangará da Serra, MT, Brasil. ORCID: 0000-0002-6624-3369 E-mail: pollyannasq@gmail.com

4. Enfermeira. Especialista em Enfermagem Obstétrica. Mestre em Enfermagem. Docente do curso de Enfermagem da UNEMAT, Campus Diamantino, Diamantino, MT, Brasil ORCID: 0000-0003-3954-8252 E-mail: helgaydoi@gmail.com

5. Enfermeira. Especialista em Gestão de Saúde Pública. Enfermeira da Estratégia Saúde da Família da Prefeitura Municipal de Sapezal, Sapezal, MT, Brasil. ORCID: 0000-0002-5401-5967 E-mail: raianerx.enfermagem@gmail.com

6. Enfermeira. Enfermeira Obstétrica. Mestre em Enfermagem. Doutoranda em Enfermagem Psiquiátrica pela Escola de Enfermagem de Ribeirão Preto, da Universidade de São Paulo. Docente do curso de Enfermagem da UNEMAT, Tangará da Serra, MT, Brasil. ORCID: 0000-0002-3901-1298 E-mail: oliveira.daniela@unemat.br 


\section{INTRODUÇÃO}

A trajetória histórica evidencia que mulheres buscam mais efetivamente por cuidados de saúde do que homens. 0 fato advém de uma construção sociocultural que atribui ao homem e a mulher papéis diferentes dentro da sociedade, conforme costumes de cada lugar, da experiência cotidiana das pessoas, bem como da maneira como se organizam a vida ${ }^{1}$.

0 conceito de cuidado precisa superar a perspectiva tradicional e buscar suprir as reais necessidades do indivíduo, ampliando as terapêuticas, aliviando sofrimentos e promovendo bem-estar ${ }^{2}$. E, em geral, os serviços públicos de saúde são espaços procurados quando se tem a necessidade de cuidado.

Com um olhar para essa crescente, o Ministério da Saúde elaborou, em 1984, o Programa de Assistência Integral à Saúde da Mulher (PAISM), um marco para a população feminina. 0 sistema de informações hospitalares da rede pública nacional aponta que o maior índice de internação hospitalar comumente se dá por mulheres ${ }^{3}$, dados que remetem a necessidade de fortalecimento dos programas e ações que visam atenção à saúde da mulher.

A atenção integral à saúde da mulher refere-se ao conjunto de ações de promoção, proteção, assistência e recuperação da saúde, executadas nos diferentes níveis de atenção à saúde sendo eles: primário, secundário e terciário. No primário, estão as Unidades Básicas de Saúde (UBS) e Estratégia de Saúde da Família (ESF), consideradas a "porta de entrada" ao Sistema Único de Saúde (SUS), em que são marcados e realizados exames, procedimentos de menor complexidade e consultas ${ }^{4}$. O secundário é entendido como de média complexidade, como as Unidades de Pronto Atendimento e Hospitais Escolas, que oferecem alguns procedimentos de intervenção, bem como tratamentos a casos crônicos e agudos de doenças. E o terciário é considerado de alta complexidade e incluem hospitais de grande porte, que possuem maior estrutura médico-hospitalar e onde são realizadas manobras mais invasivas 4 .

0 acesso aos serviços de saúde do SUS tem sido um problema estudado por várias áreas da saúde coletiva. É sabido que há uma deficiência na oferta/demanda desses serviços em todo o país. Essa dificuldade está diretamente relacionada a superlotação de unidades de maior complexidade, normalmente com demandas que poderiam ser resolvidas em unidades que se apropriam de tecnologias leve ou leve-duras, entretanto, o não atendimento das reais necessidades em saúde nas UBS faz com que a população busque a instituição hospitalar ${ }^{5}$.

Considerando o exposto, o estudo busca responder aos seguintes questionamentos: Qual o perfil sócio demográfico de mulheres que buscam cuidados no pronto atendimento de um hospital público? Qual a relação entre a utilização de um pronto atendimento de um hospital público e o atendimento na atenção básica? Assim, tem-se como objetivo descrever a busca por cuidado em um pronto atendimento de um hospital público por mulheres e a sua relação com o atendimento na atenção básica em saúde.

\section{MÉTODO}

Trata-se de um estudo de abordagem quantitativa do tipo descritivo-transversal, recorte de uma pesquisa matricial, na qual foi construído um banco de dados com informações de questionários estruturados respondidos por usuários dos serviços públicos de saúde.

Os questionários elaborados pelos pesquisadores foram aplicados durante os meses de abril e maio de 2016, em dias consecutivos e em três períodos (matutino, vespertino e noturno) em um hospital público municipal de Tangará da Serra, Mato Grosso. Para garantir rigor metodológico do banco de dados, realizou-se dupla digitação dos questionários com pareamento dos dados.

Este estudo incluiu mulheres que responderam ao questionário estruturado. Foram coletadas as seguintes variáveis de interesse: 1) Sociodemográficas: faixa etária, sexo, escolaridade, estado civil e renda familiar; 2) Relacionadas ao atendimento na atenção básica: se é cadastrada em uma UBS; se recebe visita domiciliar de profissionais da UBS e com qual 
frequência; frequência que vai a unidade em que é cadastrada; se participa de alguma atividade realizada na UBS; se faz tratamento de alguma doença crônica pelo SUS e se teve dificuldade em agendar consultas pelo SUS; 3) Relacionadas ao atendimento no hospital: motivo da procura pelo pronto atendimento hospitalar; se foi a primeira vez que procurou pelo local; se antes de ir ao hospital procurou outro serviço e qual serviço procurou.

A análise de dados se deu por meio de software estatístico, utilizando-se a análise descritiva dos resultados, apresentados por meio de frequências relativas e absolutas.

O projeto matricial intitulado "Experiência de busca por cuidado do usuário dos serviços públicos de saúde em Tangará da Serra, Mato Grosso" foi aprovado pelo Comitê de Ética em Pesquisa com Seres Humanos da Universidade do Estado de Mato Grosso, com parecer

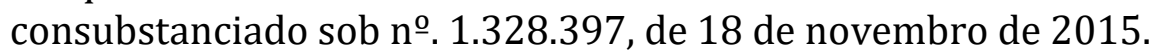

\section{RESULTADOS}

Das 181 participantes, a maior parte pertencia à faixa etária entre 36 e 59 anos de idade $(38,1 \%)$, casadas $(49,8 \%)$, ensino médio completo $(27,1 \%)$ e renda menor que dois salários mínimos $(39,7 \%)$ (Tabela 1$)$.

Tabela 1. Mulheres segundo características sociodemográficas. Tangará da Serra, Mato Grosso, 2017.

\begin{tabular}{|c|c|c|}
\hline Variáveis & Frequência (n) & Porcentagem (\%) \\
\hline \multicolumn{3}{|l|}{ Faixa etária } \\
\hline $18-24$ anos & 57 & 31,5 \\
\hline $25-35$ anos & 55 & 30,4 \\
\hline $36-59$ anos & 69 & 38,1 \\
\hline \multicolumn{3}{|l|}{ Estado civil } \\
\hline Casada & 90 & 49,8 \\
\hline Solteira & 58 & 32,0 \\
\hline Divorciada & 10 & 5,5 \\
\hline União estável & 10 & 5,5 \\
\hline Viúva & 03 & 1,7 \\
\hline Não informado & 10 & 5,5 \\
\hline \multicolumn{3}{|l|}{ Anos de estudo } \\
\hline Não estudou & 03 & 1,7 \\
\hline Sabe ler e escrever & 03 & 1,7 \\
\hline Fundamental incompleto & 37 & 20,4 \\
\hline Fundamental completo & 21 & 11,6 \\
\hline Ensino médio incompleto & 31 & 17,1 \\
\hline Ensino médio completo & 49 & 27,1 \\
\hline Superior incompleto & 23 & 12,7 \\
\hline Superior completo & 11 & 6,0 \\
\hline Não informado & 03 & 1,7 \\
\hline \multicolumn{3}{|l|}{ Renda familiar } \\
\hline Não sabe informar & 03 & 1,7 \\
\hline < 1 salário mínimo & 47 & 26,0 \\
\hline < 2 salários mínimos & 72 & 39,7 \\
\hline < 3 salários mínimos & 42 & 23,2 \\
\hline > 4 salários mínimos & 07 & 3,9 \\
\hline Não informado & 10 & 5,5 \\
\hline TOTAL & 181 & 100,0 \\
\hline
\end{tabular}

A maior parte das mulheres afirmou procurar o pronto atendimento hospitalar por manifestações clínicas relacionadas a doenças infecciosas $(23,2 \%)$, não era a primeira vez que buscava por atendimento nessa instituição $(84,5 \%)$ e que antes de buscar pelo pronto atendimento não esteve em outro serviço de saúde $(64,1 \%)$. Dentre as que buscaram outro serviço de saúde antes de procurar o hospital, a maioria $(81,6 \%)$ relatou ter buscado a UBS (Tabela 2). 
Tabela 2. Mulheres conforme atendimento no pronto atendimento hospitalar. Tangará da Serra, Mato Grosso, 2017.

\begin{tabular}{lcc}
\hline Variáveis & Frequência (n) & Porcentagem (\%) \\
\hline Motivo que procurou hospital & & \\
Doenças infecciosas & 42 & 23,2 \\
Doença digestiva/gastrintestinal & 23 & 12,7 \\
Outros (alergia, dor de dente, e outros) & 22 & 12,2 \\
Algia musculoesquelético & 16 & 8,8 \\
Doenças respiratórias & 15 & 8,2 \\
Causas externas & 14 & 7,7 \\
Gravidez/parto/puerpério & 14 & 7,7 \\
Doenças renais/geniturinárias & 13 & 7,2 \\
Doenças cardiovasculares & 13 & 7,2 \\
Encaminhada pela UBS & 4 & 2,2 \\
Transtornos mentais & 2 & 1,1 \\
Neoplasias & 1 & 0,6 \\
Alterações na pele & 1 & 0,6 \\
Não informado & 1 & 0,6 \\
1a vez que veio ao hospital & & 15,5 \\
Sim & 28 & 84,5 \\
Não & 153 & 35,9 \\
Antes de vir ao hospital procurou outro serviço & & 64,1 \\
Sim & 65 & $\mathbf{1 0 0 , 0}$ \\
Não & 116 & \\
ToTAL & $\mathbf{1 8 1}$ & \\
\hline
\end{tabular}

A maioria referiu ser cadastrada em uma UBS (89,5\%), receber visita domiciliar (63\%), não participar de nenhuma atividade promovida pela atenção primária à saúde $(96,6 \%)$, não fazer tratamento para doenças crônicas $(79,6 \%)$ e não ter dificuldade em agendar consulta pelo SUS (52\%). Das participantes cadastradas, a maior parte frequenta a UBS uma vez a cada seis meses $(40,3 \%)$ (Tabela 3$)$.

Tabela 3. Mulheres de acordo atendimento na Atenção Básica. Tangará da Serra, Mato Grosso, 2017.

\begin{tabular}{|c|c|c|}
\hline Variáveis & Frequência (n) & Porcentagem (\%) \\
\hline \multicolumn{3}{|c|}{ É cadastrada na UBS } \\
\hline Sim & 162 & 89,5 \\
\hline Não & 17 & 9,4 \\
\hline Não informado & 2 & 1,1 \\
\hline \multicolumn{3}{|c|}{ Recebe visita de alguém da UBS } \\
\hline Sim & 102 & 63,0 \\
\hline Não & 57 & 35,1 \\
\hline Não informado & 3 & 1,9 \\
\hline \multicolumn{3}{|c|}{ Frequência que vai a UBS } \\
\hline Todo dia & 2 & 1,1 \\
\hline $1 \mathrm{x}$ por semana & 9 & 5,0 \\
\hline $1 \mathrm{x}$ por mês & 48 & 26,5 \\
\hline $1 \mathrm{x}$ a cada 6 meses & 73 & 40,3 \\
\hline Nunca foi & 13 & 7,2 \\
\hline Raramente & 11 & 6,1 \\
\hline Não informado & 25 & 13,8 \\
\hline \multicolumn{3}{|c|}{ Participa de alguma atividade na UBS } \\
\hline Sim & 3 & 1,7 \\
\hline Não & 175 & 96,6 \\
\hline Não informado & 3 & 1,7 \\
\hline \multicolumn{3}{|c|}{ Faz tratamento de alguma doença crônica pelo SUS } \\
\hline Sim & 36 & 19,8 \\
\hline Não & 144 & 79,6 \\
\hline Não informado & 1 & 0,6 \\
\hline \multicolumn{3}{|c|}{ Teve dificuldade em agendar consulta pelo SUS } \\
\hline Sim & 79 & 43,6 \\
\hline Não & 94 & 52,0 \\
\hline Não informado & 8 & 4,4 \\
\hline TOTAL & 181 & 100,0 \\
\hline
\end{tabular}




\section{DISCUSSÃO}

No que se refere ao estado civil, outra pesquisa sobre a caracterização do perfil das emergências clínicas em pronto-atendimento está de acordo com os dados aqui evidenciado, na qual as mulheres eram casadas ${ }^{6}$.

Foram encontradas divergências no que diz respeito à faixa etária, prevalecendo a idade entre 19 a 25 anos em outra pesquisa ${ }^{7}$, ou seja, a maior procura pelo pronto atendimento se deu pelo público adulto jovem. É provável que o fato pode estar relacionado aos costumes de cada região no país, às experiências dos indivíduos que buscam por cuidados em saúde, e também à forma como os serviços de saúde se organizam para o atendimento da população.

Um levantamento realizado pelo Ministério da Saúde em parceria com Instituto Brasileiro de Geografia e Estatística (IBGE) abordou a porcentagem dos brasileiros que tem os serviços públicos de saúde como referência e revelou que $41,4 \%$ da população que busca por estes serviços possuem apenas o ensino fundamental incompleto 8 .

Em pesquisa sobre o grau de satisfação de usuários dos serviços de saúde quanto ao atendimento utilizado, também demonstrou que a maioria dos indivíduos possuía baixa renda . Fato que enfatiza que a situação econômica e outras características sociodemográficas são determinantes importantes para o uso dos serviços públicos de saúde.

Atualmente, algumas causas de morbimortalidade feminina prevalecem no país, como as doenças do aparelho circulatório e as neoplasias que representam a segunda maior proporção de óbitos em mulheres, destacando-se o câncer de mama e o câncer do colo do útero ${ }^{10}$. Contudo, as doenças infecciosas ainda aparecem, de acordo com os achados dessa investigação, como o principal motivo que levam mulheres a procurar o pronto atendimento hospitalar.

Possivelmente, as doenças infecciosas podem estar relacionadas às condições de vida e aos hábitos diários de mulheres e famílias no contexto do estudo, como habitação, alimentação e higiene precárias. Além disso, na época da coleta dos dados, havia a sazonalidade do Zika vírus, que também afetou o município pesquisado.

Dentre as ações e serviços protocolados nacionalmente para o combate ao vírus, o destaque está nas medidas preventivas e de combate ao mosquito transmissor, principalmente diante a possibilidade da infecção da população feminina em idade fértil, e a associação entre a microcefalia e a exposição ao vírus durante a gestação ${ }^{10}$. Destarte, a adoção de medidas preventivas assertivas e de combate ao vetor transmissor está correlacionada com as condições e hábitos de vida dos sujeitos.

As mulheres, ao declararem que não era a primeira vez que buscavam por atendimento hospitalar, enfatizaram as necessidades em saúde dessa população e a preferência por esse nível de atenção, ao relatarem majoritariamente que não estiveram em outro serviço de saúde antes de se deslocarem para a o pronto atendimento do hospital. Uma pesquisa realizada nos Estados Unidos demonstrou um aumento de 18,4\% das visitas as emergências, superando o crescimento populacional daquele país, vindo de encontro com os dados apresentados aqui, em que ainda há uma demanda por cuidados agudos e não programados ${ }^{11}$. Em algumas regiões do Brasil, como a do presente estudo essas demandas podem não estar sendo absorvidas adequadamente pela Atenção Primária à Saúde (APS).

Os gestores do SUS não devem apenas focar na oferta de serviços, mas em estratégias para que a população de fato busque os serviços preventivos e que estes sejam resolutivos baseados em necessidades coletivas e individuais ${ }^{12}$. Para isso, faz-se necessário conhecer as características e necessidades dos sujeitos que demandam por cuidado, não somente vinculado a patologias, como também aquelas relacionadas a condições de vida, às suas relações com a comunidade e aos serviços de saúde, e às percepções de como concebem e dão sentido ao processo saúde-doença.

Neste aspecto, uma investigação apontou sobre acolhimento e classificação de risco em serviços de urgência/emergência, destacando que os pacientes com problemas crônicos ou de menor complexidade deveriam ser atendidos nas unidades de atenção primária, porém por 
diversos motivos como dificuldade de acesso, ausência de profissional de saúde, insumos, entre outros, acabam buscando o pronto atendimento ${ }^{13}$.

Quando as mulheres buscam serviço de atenção à saúde de maior complexidade, há congestionamento da demanda hospitalar por causas que poderiam ser facilmente resolvidas em unidades da atenção primária. Uma realidade que poderia ser aplicada no contexto dessa investigação e em cenários semelhantes.

No tocante das variáveis que mostram o atendimento hospitalar em detrimento da atenção básica, pode ser justificado pelo fato relatado de não serem cadastradas em uma UBS e, portanto, não participarem de nenhuma atividade ofertada pela APS, bem como não fazerem nenhum tratamento para doenças crônicas, frequentando a unidade a cada seis meses.

Uma pesquisa que corrobora esse cenário mostrou que as mulheres tiveram dificuldades de acesso às UBS devido a organização da mesma, que estabelece regras para agendamento de consultas por falta de vagas, por dificuldade de comunicação das mulheres com os trabalhadores da saúde e também pela a escassez das atividades educativas, justificando a baixa frequência, e não aproximação e participação efetiva na USF14.

As mulheres participantes deste estudo não fazem tratamento de doenças crônicas pela ausência da condição crônica instalada. Em contraposição, outra pesquisa sobre as principais características dos usuários de saúde da APS no Brasil indicou que a maioria dos indivíduos participantes apresentavam doenças crônicas, sendo as doenças cardiovasculares as mais prevalentes ${ }^{15}$.

No cuidado aos usuários dos serviços de saúde, além das doenças crônicas, é necessário superar a fragmentação da atenção e da gestão em saúde e aperfeiçoar o funcionamento político institucional do SUS, com vistas a assegurar ao indivíduo o conjunto de ações e serviços que necessita com efetividade e eficiência ofertada para a este público alvo ${ }^{16}$.

A ESF é a principal organização e expansão da Atenção Básica de Saúde (ABS), por ser o ponto de atenção com maior capilaridade e potencial para identificar as necessidades de saúde da população e realizar a estratificação de riscos que subsidiará a organização do cuidado em toda a rede. É responsável também por realizar ações de promoção e de proteção da saúde, prevenção de agravos, diagnóstico, tratamento, reabilitação, redução de danos e manutenção da saúde. Além disso, a organização do processo de trabalho ofertado pela ESF se dá pelo acolhimento e acionamento de redes internas, externas e multidisciplinares ${ }^{17}$.

Por sua vez, a educação em saúde na APS é um dispositivo potente para estimular o autocuidado, a mudança de atitudes e da utilização dos serviços quanto dos comportamentos de busca de cuidados ${ }^{18}$. A coletividade gera sujeitos ativos e espaço em que as pessoas podem superar suas dificuldades e obter maior autonomia, e também estreitar a relação entre a equipe multiprofissional e o usuário, fortalecendo o vínculo e a aliança terapêutica ${ }^{17,18}$.

Portanto, a APS, como principal porta de entrada para o SUS, deveria englobar um conjunto de serviços que atendam às necessidades da população feminina e ser efetivamente resolutiva. Entretanto ainda se encontram fragilidades nesse contexto, como aqui apontado.

\section{CONCLUSÃO}

As participantes procuraram o hospital por manifestações clínicas relacionadas a doenças infecciosas, não sendo a primeira vez que buscavam por atendimento nesse serviço e a maioria não buscou outro serviço de saúde antes da procura pelo pronto atendimento hospitalar.

Apesar de a maioria ser cadastrada em uma UBS e receber visita domiciliar, as mulheres não participam de nenhum tipo de atividade na atenção primária, não fazem tratamento para doenças crônicas e não possuem dificuldade em agendar consulta pelo SUS, procurando a UBS uma vez a cada seis meses.

Nota-se a importância da efetivação de estratégias para uma maior procura feminina pelo atendimento na UBS e vínculo na atenção primária por parte dessa população, evitando grandes demandas pelos serviços secundários e terciários de saúde. 
Aponta-se como limitações do estudo o desenho transversal, o qual não permite identificar as relações de causalidade entre as variáveis. Além da não utilização de questionários validados, entretanto, os questionários foram construídos pelos pesquisadores para investigar demandas locais e específicas da população estudada e atendeu aos objetivos dessa investigação.

A realização de pesquisas que contemplem a temática aqui abordada são fundamentais para conhecer o perfil da população feminina atendida em serviços públicos, fornecendo dados sólidos para criação de ações que fortaleçam o vínculo de mulheres com a atenção primária à saúde, diminuído a superlotação dos outros níveis de atenção à saúde pública.

Sugere-se ainda realização de novos estudos sobre a temática, haja vista que os serviços de saúde e seus gestores estão em constante mudança. Incentiva-se a elaboração e a manutenção de estratégias para o adequado funcionamento da rede de saúde pública e seus níveis de atenção, para o atendimento da população feminina no país.

\section{REFERÊNCIAS}

1. Alves BM, Pitanguy J. 0 que é feminismo. São Paulo: Abril cultural, Brasiliense; 1985. 77p.

2. Kalichiman AO, Ayres JRCM. Integralidade e tecnologias de atenção à saúde: uma narrativa sobre contribuições conceituais à construção do princípio da integralidade no SUS. Cad Saúde Públ. [Internet]. 2016 [citado em 13 jul 2020]; 32(8):e00183415. DOI: https://doi.org/10.1590/0102-311X00183415

3. Ministério da Saúde (Br), Departamento de Informática do SUS. Informações de saúde. Informações epidemiológicas e morbidade. Morbidade Hospitalar do SUS (SIH/SUS): internações segundo sexo: 2020 [Internet]. [Brasília, DF: DATASUS; 2020] [citado em 16 jul 2020]. Disponível em: http://tabnet.datasus.gov.br/cgi/tabcgi.exe?sih/cnv/nrbr.def

4. Ministério da Saúde (Br), Secretaria de Gestão do Trabalho e da Educação na Saúde, Departamento de Gestão da Educação na Saúde. Política de educação e desenvolvimento para o SUS: caminhos para a educação permanente em saúde - pólos de educação permanente em saúde [Internet]. Brasília: MS; 2004 [citado em 15 dez 2016]. Disponível em: https://bvsms.saude.gov.br/bvs/publicacoes/politica2_vpdf.pdf

5. Marques AP, Montilla DER, Almeida WS, Andrade CLT. Condições sensíveis na internação de idosos. Rev Saúde Públ. [Internet]. 2014 [citado em 15 dez 2016]; 48(5):817-26. DOI: 10.1590/S0034-8910.201404800513

6. Nascimento SS. Diagnóstico de enfermagem em uma unidade de pronto atendimento: utilizando os sistemas de King [Internet]. dissertação. Brasília, DF: Universidade de Brasília; 2017 [citado em 02 out 2020]. 163p. Disponível em: https://repositorio.unb.br/bitstream/10482/24785/1/2017_SimoneSouzaNascimento.pdf

7. Hertel VL, Guimarães KS, Cardoso AO. Perfil sócio demográfico e clínico dos usuários de um pronto socorro de uma instituição do Vale do Paraíba. Rev Ciênc Saúde [Internet]. 2017 [citado em 12 jan 2018]; 7(2): 10-15. DOI: 10.21876/rcsfmit.v7i2.662

8. Ministério da Saúde (Br). Pesquisa Nacional de Saúde. Portal Brasil: 71\% dos brasileiros tem serviços públicos de saúde como referência [Internet]. Brasília, DF: Ministério da Saúde; 2015 [citado em 12 jan 2018]. Disponível em: www.brasil.gov.br/saúde/2015-serviços-publicoscomo-referencia

9. Moimaz SAS, Marques JAM, Saliba O, Garbin CAS, Zina LG, Saliba NA. Satisfação e percepção do usuário do SUS sobre o serviço público de saúde. Physis [Internet]. 2010 [citado em 20 dez 2016]; 20(4):1419-40. DOI: https://doi.org/10.1590/S0103-73312010000400019

10. Ministério da Saúde (Br). Mortalidade feminina. Informações de saúde [Internet]. Brasília, DF: Ministério da Saúde; 2013 [citado em 02 jan 2018]. Disponível em: http://www.brasil.gov.br/saude/2012/10/mortalidade-feminina-no-brasil-cai-12 
11. Lin MP, Baker O, Richardson LD, Schuur JD. Trends in emergency department visits and admission rates among US acute care hospitals. JAMA Intern Med. [Internet]. 2018 [citado em 14 jul 2020]; 178(12):1708-10. DOI: http://10.1001/ jamainternmed.2018.4725

12. Ministério da Saúde (Br), Secretaria de Vigilância em saúde. Vírus Zika no Brasil: a resposta do SUS. Brasília, DF: MS; 2017 [citado em 02 jan 2018]. Disponível em: https://bvsms.saude.gov.br/bvs/publicacoes/virus_zika_brasil_resposta_sus.pdf

13. Mendes EV. A construção social da atenção primaria a saúde [Internet]. Brasília, DF: CONASS; 2015 [citado em 20 dez 2016]. Disponível em: https://www.conass.org.br/biblioteca/pdf/A-CONSTR-SOC-ATEN-PRIM-SAUDE.pdf

14. Camargo Neto O, Andrade GKS, Karpiuck LB, Ganassin AR. A atuação do enfermeiro no sistema de acolhimento e classificação de risco nos serviços de saúde. J Health Sci. [Internet]. 2018 [citado em 13 jul 2020]; 20(4):295-302. DOI: http://dx.doi.org/10.17921/24478938.2018v20n4p295-302

15. Araújo AJS, Oliveira JF, Porto PN. Situações de vulnerabilidade programática vivenciadas por gestantes no pré-natal. Rev Enferm Atenção Saúde [Internet]. 2017 [citado em 02 jan 2018]; 6(2):93-104. DOI: 10.18554/reas.v6i2.2092

16. Guibu IA, Moraes JC, Guerra Junior AA, Costa EA, Acurcio FA, Costa KS, et al. Características principais dos usuários dos serviços de atenção primária à saúde no Brasil. Rev Saúde Pública [Internet]. 2017 [citado em 13 jul 2020]; 51(Supl 2):17s. DOI: https://doi.org/10.11606/S1518-8787.2017051007070

17. Ministério da Saúde (Br). Portaria MS/GM no 4.279, de 30 de dezembro de 2010. Estabelece as diretrizes para a organização da rede de atenção à saúde no âmbito do Sistema Único de Saúde [Internet]. Brasília, DF: MS; 2010. Disponível em: https://bvsms.saude.gov.br/bvs/saudelegis/gm/2010/prt4279_30_12_2010.html

18. Metwally AM, Abdel-Latif GA, Mohsen A, Etreby LE, Elmosalami DM, Saleh RM, et al. Strengths of community and health facilities based interventions in improving women and adolescents' care seeking behaviors as approaches for reducing maternal mortality and improving birth outcome among low income communities of Egypt. BMC Health Serv Res. [Internet]. 2020 [citado em 14 jul 2020]; 20:592. DOI: 10.1186/s12913-020-05412-1 


\section{AGRADECIMENTOS}

Ao grupo de pesquisa Laboratório de Investigação Científica em Enfermagem e Saúde (LINCES) da Universidade do Estado de Mato Grosso, pela contribuição na coleta de dados. E, ao Prof. Dr. Leandro Felipe Mufato pela idealização do projeto matricial.

\section{CONTRIBUIÇÕES}

Thais Renata Ribeiro de Souza participou da concepção, coleta e análise dos dados e redação. Denize Jussara Rupolo Dall'Agnol, Pollyanna de Siqueira Queirós e Helga Yuri Doi participaram da redação e revisão. Raiane Rocha Xavier atuou da coleta e análise dos dados. Daniela do Carmo Oliveira Mendes contribuiu na concepção, coleta e análise dos dados, redacão e revisão.

\section{Como citar este artigo (Vancouver)}

Souza TRR, Dal'Agnol DJR, Queirós PS, Doi HY, Xavier RR, Mendes DCO. Busca por cuidado em pronto atendimento público pela população feminina. REFACS [Internet]. 2020 [citado em inserir dia, mês e ano de acesso]; 8(Supl. 3):1000-1008. Disponível em: inserir link de acesso. DOI: inserir link do DOI.

\section{Como citar este artigo (ABNT)}

SOUZA, T. R. R.; DAL'AGNOL, D. J. R.; QUEIRÓS, P. S.; DOI, H. Y.; XAVIER, R. R.; MENDES, D. C. O. Busca por cuidado em pronto atendimento público pela população feminina. REFACS, Uberaba, MG, v. 8, p. 1000-1008, 2020. Supl. 3. Disponível em: inserir link de acesso. Acesso em: inserir dia, mês e ano de acesso. DOI: inserir link do DOI.

\section{Como citar este artigo (APA)}

Souza, T.R.R., Dal'Agnol, D.J.R., Queirós, P.S., Doi, H.Y., Xavier, R.R., \& Mendes, D.C.O. (2020). Busca por cuidado em pronto atendimento público pela população feminina. REFACS, 8(Supl. 3), 1000-1008. Recuperado em inserir dia, mês e ano de acesso de inserir link de acesso. DOI: inserir link do DOI. 